\title{
Identification and Pharmacological Analysis of High Efficacy Small Molecule Inhibitors of EGF-EGFR Interactions in Clinical Treatment of Non-Small Cell Lung Carcinoma: a Computational Approach
}

\author{
Suresh Gudala ${ }^{1}$, Uzma Khan ${ }^{2,3}$, Niteesh Kanungo², Srinivas Bandaru1, Tajamul \\ Hussain $^{4}$, MS Parihar ${ }^{3}$, Anuraj Nayarisseri ${ }^{2 *}$, Hema Prasad Mundluru ${ }^{1}$
}

\begin{abstract}
Inhibition of EGFR-EGF interactions forms an important therapeutic rationale in treatment of non-small cell lung carcinoma. Established inhibitors have been successful in reducing proliferative processes observed in NSCLC, however patients suffer serious side effects. Considering the narrow therapeutic window of present EGFR inhibitors, the present study centred on identifying high efficacy EGFR inhibitors through structure based virtual screening strategies. Established inhibitors - Afatinib, Dacomitinib, Erlotinib, Lapatinib, Rociletinib formed parent compounds to retrieve similar compounds by linear fingerprint based tanimoto search with a threshold of $90 \%$. The compounds (parents and respective similars) were docked at the EGF binding cleft of EGFR. Patch dock supervised protein-protein interactions were established between EGF and ligand (query and similar) bound and free states of EGFR. Compounds ADS103317, AKOS024836912, AGN-PC-0MXVWT, GNF-Pf-3539, SCHEMBL15205939 were retrieved respectively similar to Afatinib, Dacomitinib, Erlotinib, Lapatinib, Rociletinib. Compound-AGN-PC-0MXVWT akin to Erlotinib showed highest affinity against EGFR amongst all the compounds (parent and similar) assessed in the study. Further, AGN-PC-0MXVWT brought about significant blocking of EGFR-EGF interactions in addition showed appreciable ADMET properties and pharmacophoric features. In the study, we report AGN-PC-0MXVWT to be an efficient and high efficacy inhibitor of EGFR-EGF interactions identified through computational approaches.
\end{abstract}

Keywords: Non-small cell lung carcinoma - EGFR inhibitors - molecular docking - virtual screening

Asian Pac J Cancer Prev, 15 (18), 8191-8196

\section{Introduction}

Non-small cell lung carcinoma forms one of the most common cancers in the world in terms of both incidence and mortality (Ferlay et al., 2010) and one among of the leading causes of deaths in India (Takiar et al., 2010). It accounts for $13 \%$ (1.6 million) of the total cancer cases and $18 \%$ (1.4 million) of the total cancer deaths worldwide (Jemal et al., 2011).

Since the discovery of the first receptor tyrosine kinase (RTK) more than a quarter of a century ago, many members of this family of cell-surface receptors have emerged as key regulators of cell survival and metabolism, cell migration, and cell-cycle control (Blume-Jensen et al., 2001) critical cellular processes, such as proliferation and differentiation; (Ullrich et al., 1990). Among them, one particular molecular target of high promise in oncology is the epidermal growth factor receptor (EGFR). The epidermal growth factor receptor (EGFR, ErbB-1, HER1 in humans) exists on the cell surface and is activated by binding of its specific ligands, majorly including epidermal growth factor (EGF) (Usuda et al., 2013). Upon activation by its growth factor ligands, EGFR stimulates its intrinsic intracellular protein - tyrosine kinase activity which by autophophorylation events initiate several signal transduction cascades ( principally the MAPK, PTEN/Akt and JNK pathways) leading to DNA synthesis and cell division and proliferation (Wang et al., 2012).

The prognostic importance of EGFR expression in Non-Small Cell lung cancer and more importantly, its ability to predict response to anti-EGFR therapies are currently the subjects of active research. Several drugs have been developed and approved by the US Food and Drug Administration (FDA) for treating

${ }^{I}$ Institute of Genetics and Hospital for Genetic Diseases, Osmania University, Hyderabad, ${ }^{2}$ In silico Research Laboratory, Eminent Biosciences, Indore, ${ }^{3}$ School of Studies in Zoology \& Biotechnology, Vikram University, Ujjain, India, ${ }^{4}$ Center of Excellence in Biotechnology Research, College of Science, King Saud University, Riyadh, Saudi Arabia. *For correspondence: anuraj@ eminentbio.com 
cancers and other diseases caused by activated RTKs. However, the response rate to these drugs is only about $20 \%$, corresponding to an increase of three months in median survival, with no significant difference between different regimens (Marino et al., 2002). Like many other anticancer medications, targeted therapies show efficacy in only a subset of recipients. It seems that a 'therapeutic plateau' has been reached using standard cytotoxic chemotherapy, and the prognosis of advanced NSCLC remains poor (Yang 2014). Numerous side effects have been associated with the present EGFR inhibitors mainly involving dermatological diagnosis of papulopustular rash, paronychias, conjunctivitis, hypertrichosis, skin fissures, and generalized pruritus (Lynch et al., 2007; Lacouture et al., 2010). In some patients regular use of cetuximunab had complaints of electrolyte imbalances such as hypomagnesemia and hypokalemia (Thatcher et al., 2005; Lacouture et al., 2006). Hematologic side effects are generally not significant with EGFR inhibitors however; Febrile neutropenia was more significant with cetuximab and geftinib (Pirker et al., 2009). Thus, there is a continuous need for new treatments to improve survival.

Therefore in the view of given drawbacks, present study centres to identify high affinity anti-EGFR compound with a potential to disrupt the EGFR-EGF interactions in the clinical treatment of NSCLC.

\section{Materials and Methods}

Selection of inhibitors and structure similarity search

Five potential recently FDA approved EGFR inhibitors - Afatinib, Dacomitinib, Erlotinib, Lapatinib, Rociletinib served as query molecules for shape similarity search.

Similarity search, preparation of protein and compounds

The selected inhibitors served as query molecules for shape similarity search. Similarity search was supervised by binary finger print based tanimoto similarity equation to retrieve compounds with similarity threshold of 95 $\%$ against NCBI's PubChem compound database. All the structures were optimized through OPLS 2005 force field algorithm (Jorgensen et al., 1996) embedded in the LigPrep module of Schrödinger suite, 2013 (Schrodinger. LLC, New York, NY) (Schrodinger. LLC, New York, NY). Structural complex of Human EGFR and EGF was retrieved from Protein Data Bank (PDB ID: 1NQL)
(Ferguson et al, 2003) which was processed by removing all bound crystal water molecules and adding hydrogen bonds. Explicit hydrogen, bond orders, disulphide bonds, hybridizations and charges were assigned wherever missing. The resulting structure was energy minimized at protonation state of 7.4 using OPLS-2005 force field by protein preparation wizard of Schrödinger suite 2013 .

\section{Ligand receptor docking}

Molecular docking program Molegro Virtual Docker (MVD, 2010.4.0.0) which includes highly efficient PLP (Kelotra et al., 2014) and MolDock scoring function (Yang et al., 2004) provided flexible platform for molecular docking (Bandaru et al., 2015). The optimized structures of Afatinib, Dacomitinib, Erlotinib, Lapatinib, Rociletinib were docked into the active site of (EGF binding site) EGFR. Docking parameters were set to $0.20 \AA$ as grid resolution, maximum iteration of 1500 and maximum population size of 50. Energy minimization and hydrogen bonds were optimized after the docking. Simplex evolution was set at maximum steps of 300 with neighbourhood distance factor of 1 . Binding affinity and interactions of inhibitor with protein was evaluated on the basis of the internal ES (Electrostatic Interaction), internal hydrogen bond interactions and sp2-sp2 torsions. Post docked ligand-receptor complex energy was minimized using Nelder Mead Simplex Minimization (using non-grid force field and $\mathrm{H}$ bond directionality) (Nelder et al., 1965).

\section{Protein-protein docking studies}

Structural complex of Human EGFR and EGF was retrieved from Protein Data Bank (PDB ID: 1NQL). The EGFR and EGF structures were separated and saved in two different pdb files. The EGF was docked to free and ligand bound EGFR. Protein - Protein docking was executed through object recognition and image segmentation algorithm embedded in Patchdock server (Schneidman-Duhovny et al., 2005). Default parameter was set as clustering RMSD at 4.0.

\section{ADMET and bioactivity prediction of compounds.}

All the similar compounds retrieved were screened for its drug ability by lipinksi filters. The complete ADMET properties were calculated using admetSAR (Cheng et al., 2012) online webserver. Biological activity score of the ligands was predicted using Molinspiration

Table 1. MolDock Algorithm Aided Docking of Compounds in EGFR Protein. AGN-PC-OMXVWT (PubChem ID: 10048580) akin to Erlotinib shows highest affinity (rerank score) against the EGFR

\begin{tabular}{|c|c|c|c|c|c|c|}
\hline \multirow[t]{2}{*}{ Compound } & \multirow{2}{*}{$\begin{array}{l}\text { MolDock } \\
\text { Score }\end{array}$} & \multirow{2}{*}{$\begin{array}{l}\text { Rerank } \\
\text { Score }\end{array}$} & \multirow[t]{2}{*}{ Internal } & \multirow[b]{2}{*}{ H Bond } & \multicolumn{2}{|c|}{ Electrostatic range } \\
\hline & & & & & Long & short \\
\hline Afatinib & -110.209 & -72.905 & -10.875 & -1.011 & 0.252 & 0.185 \\
\hline Dacomitinib & -142.95 & -84.2 & -18.328 & -1.898 & 0.356 & 0.291 \\
\hline Erlotinib & -171.56 & -107.896 & -34.875 & -2.451 & 0.46 & 0.362 \\
\hline Lapatinib & -117.551 & -70.991 & -5.648 & -1.372 & 0.577 & 0.265 \\
\hline Rociletinib & -132.54 & -68.956 & -5.328 & -1.898 & 0.1 & 0.124 \\
\hline Afatinib similar - ADS-1033 & -152.56 & -126.647 & -28.994 & -2.5 & 0.642 & 0.554 \\
\hline Dacomitinib similar - AKOS024836912 PubChem ID: 90544772 ) & -168.829 & -114.109 & -16.923 & -2.5 & 0.436 & 0.622 \\
\hline Erlotinib similar - AGN-PC-0MXVWT (PubChem ID: 10048580 ) & -174.95 & -138.746 & -44.023 & -2.634 & 0.988 & 0.795 \\
\hline Lapatinib Simila - GNF-Pf-3539 (PubChem ID: 1285085 ) & -171.25 & -111.236 & -12.325 & -1.278 & 0.395 & 0.556 \\
\hline Rociletinib similar - SCHEMBL15205939 (PubChem ID: 89764178 ) & -166.235 & -128.234 & -38.956 & -1.354 & 0.568 & 0.612 \\
\hline
\end{tabular}


High Efficacy Small Molecule Inhibitors of EGF-EGFR Interactions in Clinical Treatment of NSCLC

webserver (@) Molinspiration Cheminformatics 2014). LC 50 was predicted using T.E.S.T. Version 4.1 (2012, U.S. Environmental Protection Agency) software.

\section{Solvent accessible surface area (SASA) and Interface Property Calculation}

Solvent accessible surface area of the complexes was calculated by GETAREA server (Ahmad et al., 2004), protein interfaces were calculated by Aquaprot (Reichmann et al., 2008) and interface property was calculated using 2P2I inspector (Basse et al., 2013) online server.

\section{Results and Discussion}

Evident from docking (rerank) scores, among the established parent compounds undertaken in the study, Erlotinib showed highest binding affinity against EGFR further, it was interesting to note that all the similar compounds identified against parent compound had higher binding affinity against EGFR protein in comparison to their respective parent compounds. Further, compound AGN-PC-0MXVWT (PubChem ID: 10048580) akin to Erlotinib showed highest affinity against the EGFR amongst all the compounds (parent and similars) undertaken in this study. Erlotinib similar- AGN-PCOMXVWT showed 1.3 folds higher affinity than its parent compound Erlotinib. The docking scores of parent and their respective similars are provided in Table 1.
In the further analysis we investigated the rationale behind the high affinity of AGN-PC-OMXVWT against EGFR. Molecular insights revealed that the internal ligand interactions of AGN-PC-OMXVWT grossly contribute for its higher affinity with EGFR interactions. As shown in table 1, the higher binding affinity of Erlotinib similar can further be attributed to higher hydrogen bonding potential along with long and short range electrostatic interaction.

The similar compounds retrieved against each parent were further tested for their in silico ADMET profile and solubility properties (Table 2). Except for SCHEMBL15205939 (PubChem ID: 89764178) a similar compound to Rociletinib the entire similar compounds retrieved, demonstrated appreciable pharmacological profile. Further, the LC50 values and bioactivity scores were quite considerable for the entire compounds screened against their parents (Table 3). Of particular note, erlotinib similar AGN-PC-0MXVWT demonstrated better LC values among all the similar compounds retrieved. In addition of having least LC 50 value, AGN-PC-0MXVWT also showed highest activity against the receptor which testifies it to be target specific. Since AGN-PC-OMXVWT shows least specificity to other receptors and showing highest specificity against receptors (in the present case Receptor Tyrosine Kinases), therefore it can be well presumed that it should activate only the receptors and not any other drug targets and thereby show least amount of side effect by not affecting the function of other drug targets.

Table 2. ADMET Prediction of Virtually Screened Compounds by ADMETSAR Server.

\begin{tabular}{|c|c|c|c|c|c|}
\hline PROPERTIES & $\begin{array}{l}\text { Afatinib similar - De } \\
\text { ADS103317 } \\
\text { (PubChem ID: } \\
\text { 44418007) }\end{array}$ & $\begin{array}{l}\text { Dacomitinib similar - } \\
\text { AKOS024836912 AG } \\
\text { (PubChem ID: } \\
\text { 90544772) }\end{array}$ & $\begin{array}{l}\text { - Erlotinib similar-L } \\
\text { GN-PC-0MXVWT } \\
\text { (PubChem ID: } \\
\text { 10048580) }\end{array}$ & $\begin{array}{l}\text { Lapatinib Similar - R } \\
\text { GNF-Pf-3539 } \\
\text { (PubChem ID: } \\
\text { 1285085) }\end{array}$ & $\begin{array}{l}\text { Rociletinib similar - } \\
\text { HEMBL15205939 } \\
\text { (PubChem ID: } \\
\text { 89764178) }\end{array}$ \\
\hline \multicolumn{6}{|l|}{ Absorption } \\
\hline Blood-Brain Barrier & BBB- & BBB- & BBB- & BBB- & BBB- \\
\hline Caco-2 Permeability & Caco2- & Caco2+ & Caco2+ & Caco2+ & Caco2- \\
\hline P-glycoprotein Substrate & Substrate & Substrate & Substrate & Substrate & Substrate \\
\hline $\begin{array}{l}\text { Renal Organic Cation Transporter } \\
\text { Distribution \& Metabolism }\end{array}$ & r Non-inhibitor & Non-inhibitor & Non-inhibitor & Non-inhibitor & Non-inhibitor \\
\hline CYP450 2C9 Substrate & Non-substrate & Non-substrate & Non-substrate & Non-substrate & Non-substrate \\
\hline CYP450 2D6 Substrate & Non-substrate & Non-substrate & Substrate & Non-substrate & Non-substrate \\
\hline CYP450 3A4 Substrate & Substrate & Substrate & Substrate & Substrate & Substrate \\
\hline \multicolumn{6}{|l|}{ Excretion \& Toxicity } \\
\hline \multicolumn{6}{|c|}{ Human Ether-a-go-go-Related Gene Inhibition } \\
\hline & Weak inhibitor & Weak inhibitor & Weak inhibitor & Weak inhibitor & Weak inhibitor \\
\hline AMES Toxicity & Non AMES toxic & ic Non-AMES toxic & Non AMES toxic & c Non AMES toxic & c AMES toxic \\
\hline Carcinogens & Non-carcinogens & is Non-carcinogens & Non-carcinogens & s Non-carcinogens & s Non-carcinogens \\
\hline Acute Oral Toxicity & III & III & III & III & III \\
\hline
\end{tabular}

*All the virtually screened compounds predicted to be safe except for Rociletinib similar compound SCHEMBL15205939 (PubChem ID: 89764178) which showed Ames toxicity

Table 3. Predicted LC 50 and Bioactivity of Similar Compounds

\begin{tabular}{|c|c|c|c|c|c|c|c|}
\hline BIOACTIVITY & $\begin{array}{l}\text { LC } 50 \\
96 \text { hour }\end{array}$ & $\begin{array}{l}\text { GPCR } \\
\text { ligand }\end{array}$ & $\begin{array}{c}\text { Ion } \\
\text { channel } \\
\text { modulator }\end{array}$ & $\begin{array}{l}\text { Kinase } \\
\text { inhibitor }\end{array}$ & $\begin{array}{c}\text { Nuclear } \\
\text { receptor } \\
\text { ligand }\end{array}$ & $\begin{array}{l}\text { Protease } \\
\text { inhibitor }\end{array}$ & $\begin{array}{l}\text { Enzyme } \\
\text { inhibitor }\end{array}$ \\
\hline Afatinib similar ADS-103317 (PubChem ID: 44418007) & 22.38 & 0.34 & -0.23 & -0.04 & -0.04 & 0.19 & 0 \\
\hline Dacomitinib similar AKOS024836912 (PubChem ID: 90544772 ) & 21.28 & -0.62 & -1.34 & -1.05 & -1 & -0.69 & -1.02 \\
\hline Erlotinib similar AGN-PC-0MXVWT (PubChem ID: 10048580 ) & 39.52 & 0.06 & -0.36 & -0.18 & 1.55 & -0.32 & -0.3 \\
\hline Lapatinib Similar GNF-Pf-3539 (PubChem ID: 1285085 ) & 13.24 & 0.04 & -0.58 & -0.14 & 0.04 & 0.08 & -0.38 \\
\hline Rociletinib similar SCHEMBL15205939 (PubChem ID: 89764178 ) & 17.23 & 0.51 & -0.71 & -1.6 & 0.02 & -1.8 & -0.11 \\
\hline
\end{tabular}


Table 4. EGFR Inhibitors Bind at the EGF Binding site of the Receptor Making EGF Inaccessible to Receptor and Thereby Imparting Reduced Interactions of EGF to the EGFR

\begin{tabular}{lccc}
\hline PROTEIN 1 & PROTEIN 2 & Score & Transformation \\
\hline EGFR bound to : & & & \\
Afatinib & EGF & 6391 & $-0.52-0.56-3.1142 .87-50.8211 .99$ \\
Dacomitinib & EGF & 6894 & $3.13-0.72-2.84-4.30-5.884 .39$ \\
Erlotinib & EGF & 6094 & 3.470 .6612 .2042 .780 .822 .44 \\
Lapatinib & EGF & 6926 & $-0.482-1.20-1.6843 .65-12.3521 .52$ \\
Rociletinib & EGF & 6979 & $-0.56-1.35-1.9928 .96-15.6825 .35$ \\
Afatinib similar ADS-103317 (PubChem ID: 44418007) & EGF & 6010 & $-0.65-1.142 .6123 .66-54.5512 .43$ \\
Dacomitinib similar AKOS024836912 (PubChem ID: 905447772) & EGF & 6115 & $-3.79-0.273 .4561 .31-29.60-16.44$ \\
Erlotinib similar AGN-PC-0MXVWT (PubChem ID: 10048580) & EGF & 5002 & $3.11-0.64-1.18-5.16-16.32-3.05$ \\
Lapatinib Similar GNF-Pf-3539 (PubChem ID: 1285085) & EGF & 5556 & $3.431 .752 .8860 .62-11.85-21.74$ \\
Rociletinib similar SCHEMBL15205939 (PubChem ID:89764178) & EGF & 5789 & $-2.350 .285 .8156 .54-12.21-22.56$ \\
EGFR Ligand unbound state & EGF & 10214 & $-2.380 .272 .0062 .28-48.79-38.45$ \\
\hline
\end{tabular}

*The table shows EGFR-EGF protein-protein docking at EGFR ligand bound and free states. The interactions of EGFR-EGF considerably decline in presence of inhibitors and their respective similars. The interactions are further declined (lowest score implies least interactions and vice-versa) in presence of Erlotinib similar AGN-PC-0MXVWT (PubChem ID: 10048580)

Table 5. Interface Property Calculations in Ligand free and Ligand Bound States EGFR-EGF Complex

\begin{tabular}{|c|c|c|c|c|c|c|}
\hline & $\begin{array}{l}\text { Total Interface } \\
\text { Area }(\AA 2)\end{array}$ & $\begin{array}{l}\text { e Gap } \\
\text { volume }(\AA 3)\end{array}$ & $\begin{array}{l}\text { Gap } \\
\text { Index }\end{array}$ & SASA & $\begin{array}{c}\text { Buried } \\
\text { atoms }\end{array}$ & $\begin{array}{c}\text { Surface } \\
\text { atoms }\end{array}$ \\
\hline \multicolumn{7}{|l|}{ EGFR bound to : } \\
\hline Afatinib & 1486 & 876 & 0.589502 & 818 & 510 & 738 \\
\hline Dacomitinib & 1514 & 826 & 0.545575 & 798 & 598 & 650 \\
\hline Erlotinib & 1302 & 912 & 0.700461 & 889 & 488 & 760 \\
\hline Lapatinib & 1214 & 785 & 0.646787 & 764 & 738 & 510 \\
\hline Rociletinib & 1685.2 & 721 & 0.427961 & 712 & 652 & 596 \\
\hline Afatinib similar ADS-103 & 1289 & 968 & 0.75097 & 924 & 425 & 823 \\
\hline Dacomitinib similar AKOS024836912 (PubChem ID: 90544772 & ) 1524 & 819 & 0.537 & 987 & 590 & 845 \\
\hline Erlotinib similar AGN-PC-0MXVWT (PubChem ID: 10048580 & $0 \quad 998$ & 1248 & 1.250501 & 1298 & 283 & 965 \\
\hline Lapatinib Similar GNF-Pf-3539 (PubChem ID: 1285085 ) & 1015 & 1146 & 1.129064 & 1210 & 308 & 940 \\
\hline $\begin{array}{l}\text { Rociletinib similar SCHEMBL15205939 } \\
\text { (PubChem ID: } 89764178 \text { ) }\end{array}$ & 1154 & 1012 & 0.87695 & 1001 & 354 & 894 \\
\hline EGFR Ligand unbound state & 1782.5 & 605 & 411 & 685 & 782 & 466 \\
\hline
\end{tabular}

As evident from protein-protein docking studies, the EGFR-EGF interactions are efficiently inhibited in presence of inhibitors as compared to ligand free EGFR, thus implying there occurs a declined EGFR-EGF interactions in presence of inhibitors. It is interesting to note that, except for compound Dacomitinib similar AKOS024836912 (PubChem ID: 90544772) all the similar compounds had superior inhibitory potential than their parent compounds. In particular, Erlotinib similar AGN-PC-0MXVWT (PubChem ID: 10048580) had highest inhibitory potential among all the compounds (parent and their respective similar). Erlotinib similar showed 1.23 folds better inhibitory potential than its parent compounds Erlotinib. The patch dock scores (proteinprotein docking scores) of EGFR-EGF interaction in presence of inhibitors and their respective akin is shown in Table 4.

The rationale behind EGFR-EGF docking results in presence and absence of inhibitors were complemented by interface property calculations (Table 5). From extensive interface properties calculated it is quite apparent that all the similar compounds had well inhibitory potential than their respective parent compound (except for Dacomitinib similar AKOS024836912, in the presence of which relatively showed high interactions of EGFREGF interactions compared to remaining similar compound ). As mentioned afore, Erlotinib similar AGN-PC-0MXVWT was superior among the undertaken compounds in preventing the EGFR-EGF interactions (as revealed from patch dock scores) and interface properties calculations further asserts this observation. The concurrence between patch dock results and EGFR-EGF interface property calculation is supported by the fact that

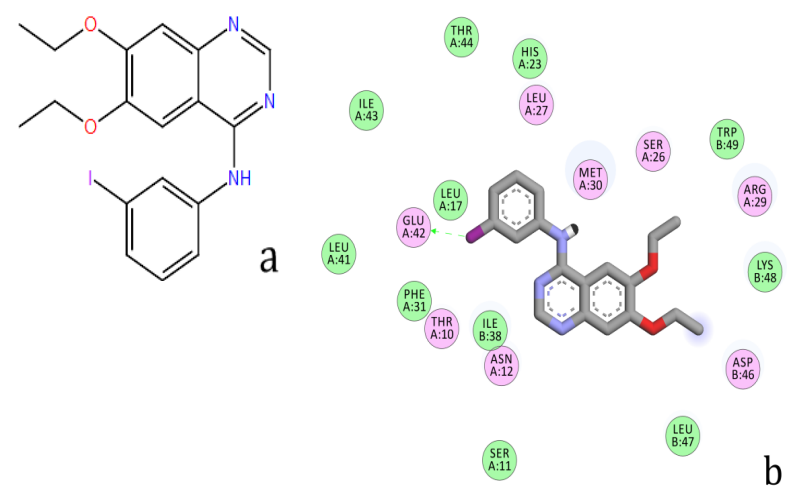

Figure 1. (a) Structure of Erlotinib Similar AGN-PC0MXVWT (PubChem ID: 10048580) (b) Interactions of AGN-PC-0MXVWT with EGFR and EGF. Residues (residues prefixed with 'A' belong to EGFR and 'B' belong to EGF). Residues circled in green participate in van der Waals interaction while residues in pink forms electrostatic interactions. Hydrogen bond vector is shown as green arrow 

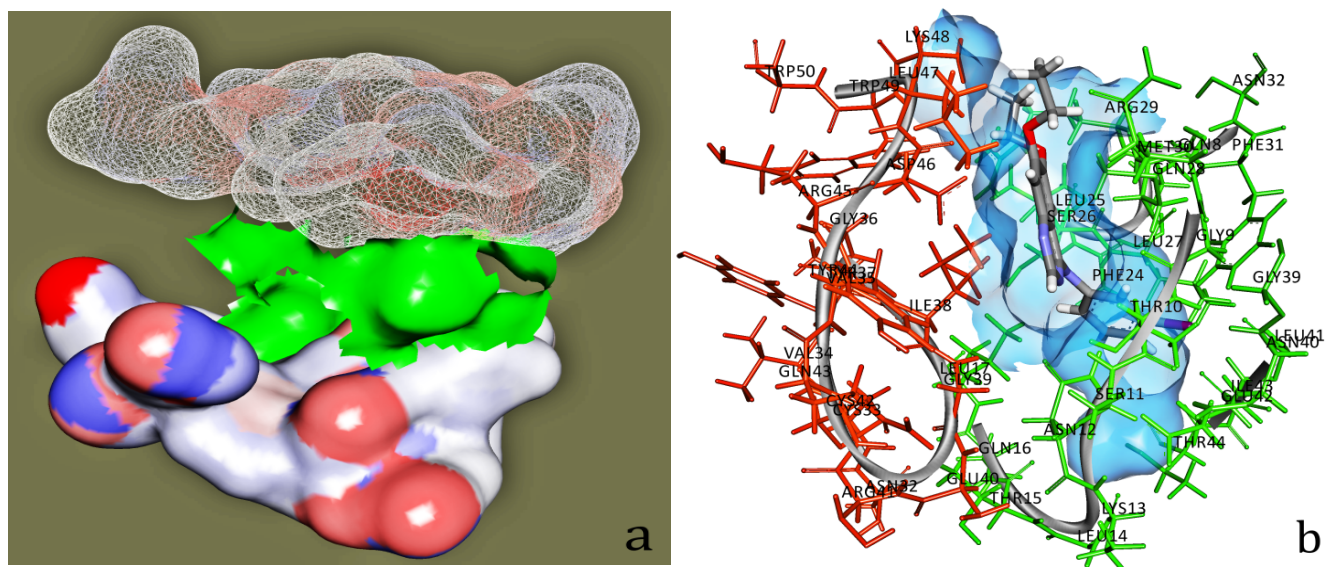

Figure 2. (a) Electrostatic Potential Surface of EGFR (Solid representation) and EGF (mesh representation). Erlotinib similar AGN-PC-0MXVWT (green solid representation) blocks the interaction of EGFR and EGF thereby preventing EGFR mediated downstream kinase signaling. (b) AGN-PC-0MXVWT at the interface (blue shade) in interaction with EGFR (green residues) and EGF (red residues)

the total interface area in EGFR-EGF complex was 1.3 folds declined in presence of Erlotinib similar AGN-PCOMXVWT compared to the complex harboring its parent compound Erlotinib. Further comparative analysis of the number of surface and buried atoms of the EGFR -EGF complexes has unveiled that AGN-PC-0MXVWT bound EGFR -EGF complex had considerable increase in the number of surface atoms and a decrease in buried atoms therefore implying a weak interaction between EGF and EGFR. In addition, AGN-PC-0MXVWT bound EGFREGF complex showed highest total solvent accessibility surface area which gives a clue towards decreased EGFREGF interactions. It is also imperative to note that the total solvent accessibility surface area of AGN-PC-0MXVWT bound EGFR-EGF complex increased by 1.4 folds when compared to the Erlotinib bound EGFR-EGF complex. Likewise, gap index was highest between EGFR-EGF in presence of AGN-PC-OMXVWT indicating it to be the powerful and potential inhibitor in disrupting EGFR-EGF interactions among the compound (Parent and respective similar) undertaken in the present study.

Owing to superior inhibitory potential of Erlotinib similar AGN-PC-0MXVWT (Figure 1a), it was further mapped for its pharmacophoric properties. As shown in Figure $1 \mathrm{~b}$, EGFR-EGF interface, and specifically at EGFR protein, compound shows van der waals interaction with Thr 44, Ile 43, Leu 41, Leu 17, Phe 31, Ser 11 and His 23 and electrostatic interactions with Leu 27, Met 30, Ser 26, Arg 29, Asn 12 and Thr 10 and H hydrogen bonding interactions with Glu 42, and in the EGF protein the compound interacts through van der waals with Lys 48, Trp 49, Ile 38 and electrostatic interactions with Asp 46. Electrostatic surface representation of EGFR-EGF in complex with AGN-PC-0MXVWT is shown in Figure 2a. Interface area and participating residues of EGFR and EGF with AGN-PC-0MXVWT is shown in Figure $2 b$.

In conclusion the together with molecular docking analysis, protein-protein patch docking, interface property and SASA analysis we put forth erlotinib akin compound AGN-PC-0MXVWT that brings about the perturbed interactions between EGFR and EGF, thereby preventing the downstream kinase signaling of EGFR associated with abrupt cell proliferation observed in NSCLC.

\section{Acknowledgements}

Funding from University Grants Commission (UGC), India for Rajiv Gandhi National Fellowship Scheme (RGNF) is highly acknowledged (Grant Number: RGNF2014-15D-OBC-AND-83567).

\section{References}

Ahmad S, Gromiha M, Fawareh H, Sarai A (2004). ASAView: database and tool for solvent accessibility representation in proteins. BMC Bioinformatics, 5, 824-27.

Bandaru S, Tiwari G, Akka J, et al (2015). Identification of high affinity bioactive salbutamol conformer directed against mutated (Thr164Ile) Beta 2 adrenergic receptor. Cur Top Med Chem, 15, 50-56.

Basse MJ, Betzi S, Bourgeas R, et al (2013). 2P2Idb: a structural database dedicated to orthosteric modulation of proteinprotein interactions. Nucleic Acids Res, 41, 824-27.

Blume-Jensen P, Hunter T (2001). Oncogenic kinase signalling. Nature, 411, 355-65.

Cheng F, Li W,Zhou Y, et al (2012). admetSAR: a comprehensive source and free tool for assessment of chemical ADMET properties. J chem inf and model, 52, 3099-105.

Dahabreh IJ, Terasawa T, Castaldi PJ, et al (2011). Systematic review: anti-epidermal growth factor receptor treatment effect modification by KRAS mutations in advanced colorectal cancer. Annal Inter Med, 154, 37-49.

Ferguson KM, Berger MB, Mendrola JM, et al (2003). EGF activates its receptor by removing interactions that autoinhibit ectodomain dimerization. Molecular Cell, 11, 507-17.

Ferlay J, Shin HR, Bray F, et al (2010). Estimates of worldwide burden of cancer in 2008: GLOBOCAN 2008. Int J Caner, 127, 2893-917.

Govindan R, Page N, Morgensztern D, et al (2006). Changing epidemiology of small-cell lung cancer in the United States over the last 30 years: analysis of the surveillance, epidemiologic, and end results database. J Clin Oncol, 24, 4539-44.

Jemal A, Bray F, Center MM, et al (2011). Global cancer statistics. CA Cancer J Clin, 61, 69-90.

Jemal A, Siegel R, Xu J, (2010). Cancer Statistics. CA Cancer 
J Clin, 60, 277-300.

Jorgensen WL, Maxwell DS, Tirado-Rives J (1996). Development and testing of the OPLS all-atom force field on conformational energetics and properties of organic liquids. J Am Chem Soc, 118, 11225-36.

Lacouture M, Basti S, Patel J, et al (2006). The SERIES Clinic: an interdisciplinary approach to the management of toxicities of EGFR inhibitors. J Support Oncol, 4, 236-8.

Lacouture ME, Maitland ML, Segaert S, et al (2010). A proposed EGFR inhibitor dermatologic adverse event-specific grading scale from the MASCC skin toxicity study group. Support Care Cancer, 18, 509-22.

LigPrep, V. (2010). 2.4, Schrödinger. LLC, New York.

Lynch TJ, Kim ES, Eaby B, et al (2007). Epidermal growth factor receptor inhibitor-associated cutaneous toxicities: an evolving paradigm in clinical management. Oncologist, 12, 610-21.

Marino P, Pampallona S, Preatoni A, et al (1994). Chemotherapy vs supportive care in advanced non-smallcell lung cancer. Results of a meta-analysis of the literature. Chest, 106, 861-5.

Nelder JA, Mead R (1965). A simplex method for function minimization. The comput $J, 7,308-13$.

Pirker R, Pereira JR, Szczesna A, et al (2009). Cetuximab plus chemotherapy in patients with advanced non-small-cell lung cancer (FLEX): an open-label randomized phase III trial. Lancet, 373, 1525-31.

Reichmann D, Phillip Y, Carmi A, Schreiber G (2008). On the contribution of water-mediated interactions to proteincomplex stability. Biochemistry, 47, 1051-60.

Schiller JH, Harrington D, Belani CP, et al (2002). Comparison of four chemotherapy regimens for advanced non-small-cell lung cancer. $N$ Engl J Med, 346, 92-8.

Schneidman-Duhovny D, Inbar Y, Nussinov R, et al (2005). PatchDock and SymmDock: servers for rigid and symmetric docking. Nucleic Acids Res, 33, 363-7

Stinchcombe TE, Bradford DS, Hensing TA, et al (2010). A multicenter phase II trial of carboplatin and cetuximab for treatment of advanced non small cell lung cancer. Cancer Invest, 28, 208-15.

Takiar R, Nadayil D, Nandakumar A (2010). Projections of number of cancer cases in India (2010-2020) by cancer groups. Asian Pac J Cancer Prev, 11, 1045-49.

Thatcher N, Chang A, Parikh P, et al (2005). Gefitinib plus best supportive care in previously treated patients with refractory advanced non-small cell lung cancer: results from a randomised, placebo-controlled, multicentre study (Iressa Survival Evaluation in Lung Cancer). Lancet, 366, 1527-37.

Ullrich A, Schlessinger J (1990). Signal transduction by receptors with tyrosine kinase activity. Cell, 61, 203-212.

Usuda K, Sagawa M, Motono N, et al (2013). Relationships between EGFR mutation status of lung cancer and preoperative factors-are they predictive? Asian Pac J Cancer Prev, 15, 657-62.

Wang YS, Wang YH, Xia H, et al (2012). MicroRNA-214 regulates the acquired resistance to gefitinib via the PTEN/ AKT pathway in EGFR-mutant cell lines. Asian Pac $J$ Cancer Prev, 13, 255-60.

Yang JM, Chen CC (2004). Gemdock: A generic evolutionary method for molecular docking. Proteins, 55, 288-304

Yang ZY, Liu L, Mao C, et al (2014). Chemotherapy with cetuximab versus chemotherapy alone for chemotherapy naive advanced non small cell lung cancer. Cochrane Database Syst Rev, 11, 9948. 